\title{
The familiarity of icy worlds
}

\author{
The geological similarities between icy and rocky worlds invite comparison and cross-fertilization of knowledge.
}

M issions to ice-rich planetary objects have revealed that these bodies are not frozen wastelands - instead, they are geologically complex and active. A variety of familiar geological phenomena have been recognized on icy worlds great and small: landslides ${ }^{1}$ and shear deformation ${ }^{2}$ have been identified on the comet 67P/Churyumov-Gerasimenko from observations by the Rosetta spacecraft, and the Cassini mission to the Saturn system found geyser-like water jets on Enceladus $^{3}$, as well as lacustrine basins ${ }^{4}$ and domes and calderas ${ }^{5}$ on Titan. The New Horizons mission discovered that the dwarf planet Pluto and its moon Charon are among the most geologically diverse icy objects ${ }^{6}$, despite their relatively cold location. Geological processes on rocky and ice-rich bodies across the Solar System may therefore be very similar ${ }^{6}$. With this issue, we present a collection of articles (https:// www.nature.com/collections/cbbhcgbjgb/) that highlight some of the latest advances in our understanding of icy worlds.

On various icy worlds - from Ceres ${ }^{7}$ in the asteroid belt to Pluto ${ }^{8}$ in the Kuiper belt - landforms have been interpreted as evidence for cryovolcanism, which entails the eruption of volatiles such as water or methane as liquid or vapour. Nevertheless, it is not clear how bodies that are not continuously heated by tidal forces can have cryovolcanic activity long after they formed. For example, on the dwarf planet Ceres, the Dawn spacecraft documented kilometreshigh domes that have been attributed to cryovolcanism. It has been proposed that Ahuna Mons, the most prominent of these domes, formed by effusion of a mixture of brine and solids sourced from a plume in a fluid, muddy mantle ${ }^{7}$. However, a News \& Views by Küppers points out that heat sources such as radiogenic material from Ceres's formation may be insufficient to maintain voluminous long-lived cryovolcanism. An Article by Bland et al. argues that at least most of the domes could alternatively be explained by a liquid-free process: solid-state flow of the relatively ice-rich parts of Ceres's crust, driven by gravitational loading, analogous to salt doming on Earth.

Conversely, planetary landforms have also been reinterpreted as cryovolcanic features based on analogy to terrestrial structures. Cassini revealed many small lakes of liquid hydrocarbons in the polar

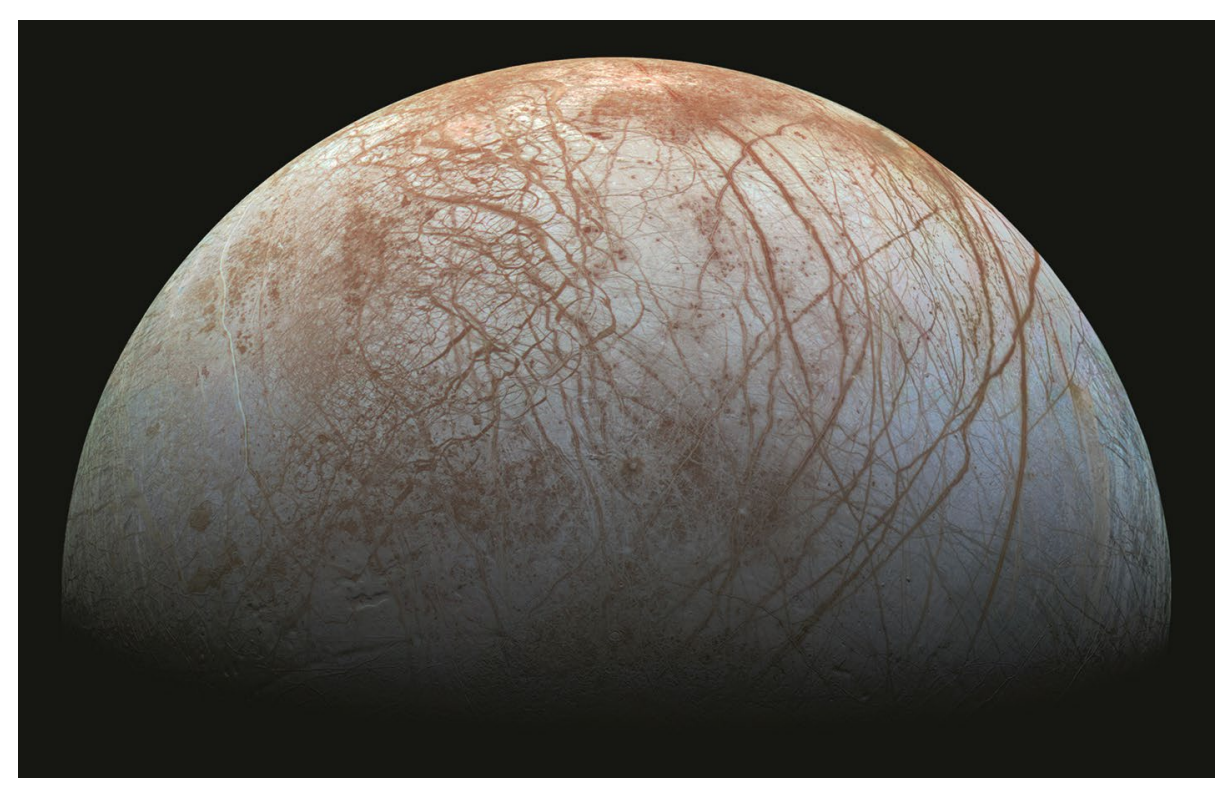

Credit: NASA/JPL-Caltech/SETI Institute

regions of Titan, particularly near its northern pole; the lake basins are thought to have formed by dissolution processes similar to those invoked in karst formation on Earth ${ }^{4}$. A subset of these basins have raised rims, however, so an Article by Mitri et al. proposes that they are instead analogous to terrestrial maars - craters from explosions due to subsurface interactions between magma and water. Mitri et al. suggest that on Titan, crater-forming explosions may be driven by the vaporization of subsurface liquid nitrogen reservoirs during past warming events.

Icy worlds are particularly interesting because some have subsurface oceans of liquid water, which may be habitable ${ }^{9}$. Even more so than for cryovolcanism, it has been unclear how such oceans can be maintained on objects without substantial heat sources. For example, on Pluto, observations by New Horizons suggest that an ocean exists beneath an ice shell ${ }^{10}$. One possible means to maintain this ocean is an insulating capping layer of clathrate (gas) hydrates, a mechanism that may be applicable to similar bodies ${ }^{11}$.

The habitability of icy worlds will be a particular focus of future missions. NASA's Europa Clipper ${ }^{12}$ and ESA's Jupiter Icy Moons Explorer (JUICE) $)^{13}$ are destined for the icy Galilean moons of Jupiter. These spacecraft will characterize the surfaces and infer the interior structures of the moons, particularly to constrain the nature of their putative subsurface oceans. On Titan, NASA's Dragonfly mission will measure the electric field to probe its possible subsurface ocean, and may target apparent cryovolcanic features to investigate their chemical interaction with organic compounds ${ }^{14}$.

As exploration of our Solar System has advanced, Earth has become less exceptional in some respects. Greater understanding of our icy neighbours provides opportunities to test our comprehension of geological processes.

Published online: 27 September 2019 https://doi.org/10.1038/s41561-019-0469-5

\footnotetext{
References

1. Pajola, M. et al. Nat. Astron. 1, 0092 (2017).

2. Matonti, C. et al. Nat. Geosci. 12, 157-162 (2019).

3. Hansen, C. et al. Science 311, 1422-1425 (2006).

4. Cornet, T. et al. J. Geophys. Res. Planets 120, 1044-1074 (2015)

5. Lopes, R. M. C. et al. Icarus 186, 395-412 (2007).

6. Schenk, P. \& Nimmo, F. Nat. Geosci. 9, 411-412 (2016).

7. Ruesch, O. et al. Nat. Geosci. 12, 505-509 (2019).

8. Cruikshank, D. P. et al. Icarus 330, 155-168 (2019).

9. Hand, K. P. \& German, C. R. Nat. Geosci. 11, 2-5 (2018).

10. Nimmo, F. et al. Nature 540, 94-96 (2016).

11. Kamata, S. et al. Nat. Geosci. 12, 407-410 (2019).

12. Phillips, C. B. \& Pappalardo, R. T. Eos 95, 165-167 (2014).

13. Dougherty, M. K. et al. EPSC Abstracts 6, EPSC-DPS2011-1343-1 (2011).

14. Lorenz, R. D. et al. J. Hopkins APL Tech. Dig. 34, 374-387 (2018).
} 